www.jmscr.igmpublication.org

Impact Factor 5.84

Index Copernicus Value: 71.58

ISSN (e)-2347-176x ISSN (p) 2455-0450

crossref DOI: _https://dx.doi.org/10.18535/jmscr/v5i10.136

Journal Of Medical Science And Clinical Research

IGM Publication

An Official Publication of IGM Publication

\title{
Retinal Vein Occlusion -Risk Factors in Younger Patients
}

\section{Authors \\ Arun Kumar Ayoor, Ranjini Kotancheri, Swathi Karuppali, Anu Bhaskar, Hasna Hussain K N}

Department of Ophthalmology, Government Medical College, Kozhikode Kerala, India Pin 673008

Corresponding Author

Dr Ranjini Kotancheri

Associate Professor of Ophthalmology, Govt Medical College, Kozhikode, Kerala India Pin 673008

Email:dr.ranjinik@gmail.com, Tel no.9349888282

\begin{abstract}
Introduction: Retinal vein occlusion (RVO) accounts for a major cause of sudden visual impairment in the population. Many of the investigations often turn out futile especially in the young and evaluation to find out the risk factors are also limited. Role of rare factors like raised serum homocysteine and vasculitis have to be kept in mind in this group and the fact that venous occlusions usually precede the development of systemic risk factors should be emphasized.

Materials and Methods: 52 patients in the age group of 11 to 50 years with a diagnosis of retinal vein occlusion were studied and classified into ischemic central retinal vein occlusion (ICRVO), non-ischemic CRVO (NICRVO), branch retinal vein occlusion (BRVO) and hemi CRVO (HCRVO).The various risk factors were analyzed including ocular risk factors, systemic hypertension, diabetes mellitus, dyslipidemia, smoking, serum creatinine and presence of hyper homocysteinemia in each age group.

Results: Mean age was 38.45 with $56 \%$ males and $42 \%$ females. Out of 52 patients analyzed, 37 were in the age group of 41 to 50, 33 had dyslipidemia, 22 patients were hypertensives, 17 were having diabetes mellitus. Serum homocysteine was elevated in 3 patients below 30 years of age and it was found to be statistically significant ( $p$ value $<0.001$ ). No association was found between cigarette smoking, high serum creatinine and RVO. Ocular risk factors present were primary open angle glaucoma in one patient and ocular hypertension in the other.

Conclusion: Detailed evaluation for systemic risk factors should be made mandatory in all cases of retinal vein occlusion. Many of the patients belonged to older age group and dyslipidemia was the major single modifiable risk factor among these. It should be kept in mind that in younger population, conventional metabolic risk factors may not be evident in the early stages and hence less common factors like high serum levels of homocysteine should be looked for and prompt treatment initiated at the earliest. Strict follow up of these patients for evaluation of any systemic diseases in the future should also be done.

Keywords: Retinal vein occlusion (RVO), branch retinal vein occlusion (BRVO), hypertension, diabetes mellitus, homocysteine, dyslipidemia, smoking, risk factors.
\end{abstract}

\section{Introduction}

Retinal vein occlusion (RVO) is the second most common cause of acquired retinal vascular abnormality in adults and frequent cause of visual loss after diabetic retinopathy. Venous occlusions are broadly classified as central retinal vein 
occlusion and branch retinal vein occlusion. Its pathogenesis is explained based on Virchow's triad: hemodynamic changes (venous stasis), degenerative changes of the vessel wall, and blood hypercoagulability. Central retinal vein occlusion (CRVO) is considered as a devastating cause of significant visual loss and is a disease predominantly of the elderly, with proven major risk factors being age, hypertension, hyperlipidemia, and diabetes mellitus. It is uncommon in younger people and is attributable to unusual causes such as hyper viscosity syndromes, hypercoagulable conditions and infectious and noninfectious vasculitic diseases. The ocular risk factor mainly identified being primary open angle glaucoma. Though this disease and its risk factors have been extensively studied in the older age groups, there are few data on the prevalence of RVO in the younger population, with current estimates of risk factors being derived largely from studies of the population as a whole rather than age specific data.

Various population-based studies report a prevalence of retinal venous occlusions varying between $0.3 \%$ and $1.6 \%$ and among that a rate of $0.5-2.0 \%$ for branch RVO and $0.1-0.2 \%$ for central $\mathrm{RVO}^{(1)}$. The 15 -year incidence rate is estimated to be $1.8 \%$ for branch RVO and $0.2 \%$ for central RVO. According to blue mountain study, the calculated prevalence among patients younger than 60 years is $0.7 \%$ which appears to be on the rise due to varying emerging risk factors and lifestyle changes. But whether the traditional established metabolic risk factors actually influence the occurrence of disease in the young is still unclear. Hence we have undertaken this study to specifically study the factors that are prevalent in the patients up to the age of 50 years who develop any form of retinal venous occlusion and also to define the investigation profile needed in newly detected case of RVO.

The main causes of visual loss after retinal venous occlusion are chronic macular edema and secondary neovascular glaucoma. Lower visionrelated quality of life was reported among patients suffering from RVO than those without ocular disease. Early diagnosis and prompt initiation of treatment either in the form of systemic factor control or invasive procedures will help in restoring vision or preventing further deterioration. Since it is a vascular event, follow up of patient for the development of any systemic complication also forms a part of the treatment regimen as it is observed that patients with RVO have a higher prevalence of stroke ${ }^{(2)}$ and a greater risk of cardiovascular disease than similarly aged individuals without $\mathrm{RVO}^{(3,4)}$.

\section{Materials and Methods}

A cross sectional study was conducted among 52 diagnosed cases of retinal venous occlusions in the age group 11 to 50 years attending the retina clinic and ophthalmology outpatient department at a tertiary ophthalmic care centre in South India during a period of 2 years from January 2014 to December 2015. The study was approved by the institutional research committee and ethical clearance was obtained. An informed written consent was taken from the study subjects.

\section{Inclusion Criteria}

All patients with recently diagnosed retinal venous occlusions (RVO) within one month in the age group of 11 to 50 years.

\section{Exclusion Criteria}

Patients with preexisting retinal and macular degenerative conditions, high myopia, retinitis pigmentosa, retinal vasculitis, combined with retinal arterial occlusions, posterior uveitis, severe nonproliferative and proliferative diabetic retinopathy and optic atrophy.

\section{Data Collection and Analysis}

After getting written informed consent, patient's data were collected. A detailed history, general and systemic examinations with blood pressure recording was done. Comprehensive ocular examination, best corrected visual acuity, slitlamp examination and intraocular pressure recorded with applanation tonometry. Fundus examination done with direct ophthalmoscope, indirect ophthalmoscope and slit lamp with +90D 
volk lens. Laboratory Investigations done included complete blood count with ESR, fasting and post prandial blood sugar values, renal function tests, fasting serum lipid profile and serum homocysteine value. ECG, medical, nephrology and cardiology consultation were done in relevant cases.

52 patients with a diagnosis of retinal venous occlusion were included in the study as per the inclusion and exclusion criteria. Data collected were analysed using SPSS software version 17.0 (SPSS, Chicago, Illinois, USA) and Chi square test was used to compare the variables.

\section{Results}

We studied a total of 52 patients, ranging from 16 to 50 years with a mean age of $38.45,29(56 \%)$ were males and 23 (42\%) females.

Fig 1. Gender distribution of the sample

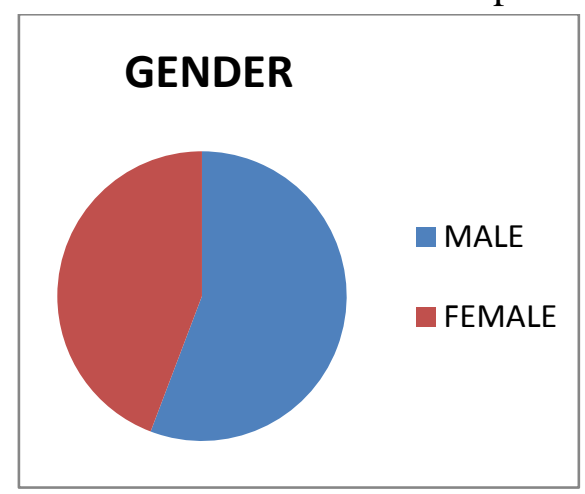

Table 1: Age group and Type of Venous occlusion

\begin{tabular}{|c|c|c|c|c|c|}
\hline \multirow{2}{*}{ Age Group } & \multicolumn{4}{|c|}{ Venous occlusion Type } & \multirow{2}{*}{ Total } \\
\cline { 3 - 6 } & ICRVO & NICRVO & HCRVO & BRVO & 1 \\
\hline $11-20$ & 1 & 0 & 0 & 0 & 4 \\
$21-30$ & 2 & 2 & 0 & 0 & 10 \\
$31-40$ & 2 & 2 & 3 & 3 & 37 \\
$41-50$ & 6 & 6 & 4 & 21 & 52 \\
Total & 11 & 10 & 7 & 24 & \\
\hline
\end{tabular}

The age groups analyzed were from 11 years to 50 years in 4 categories, out of which maximum of 37 patients belonged to the age group of 41 to 50 followed by 10 patients in the age group 31 to 40 years.

Fig 2. Eye and Venous occlusion

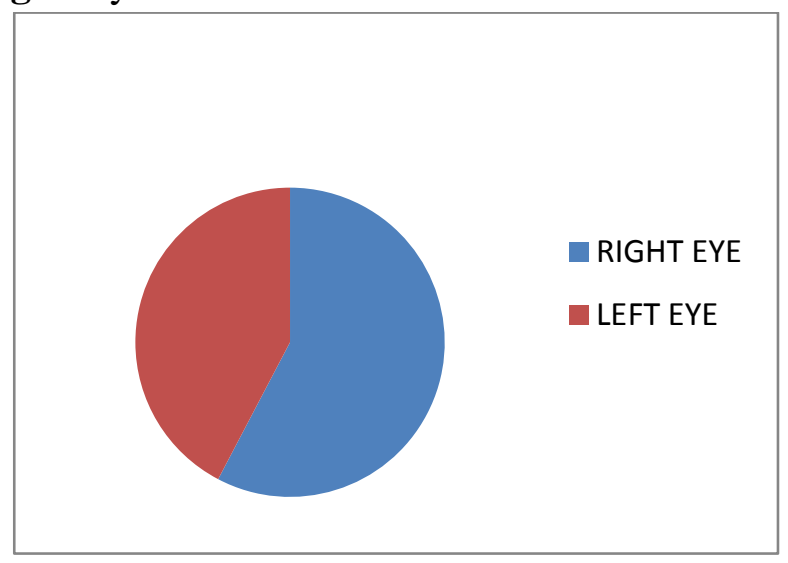

30 patients presented with venous occlusion in the right eye and 22 in the left eye

Table 2: Eye and Type of venous occlusion

\begin{tabular}{|c|c|c|c|c|c|}
\hline \multirow{2}{*}{ Eye } & \multicolumn{4}{|c|}{ VENOUS OCCLUSION TYPE } & \multirow[b]{2}{*}{ Total } \\
\hline & ICRVO & NICRVO & HCRVO & BRVO & \\
\hline RIGHT & 5 & 6 & 4 & 15 & 30 \\
\hline LEFT & 6 & 4 & 3 & 9 & 22 \\
\hline Total & 11 & 10 & 7 & 24 & 52 \\
\hline
\end{tabular}

Majority of the patients presented with venous occlusion in the right eye (30), right and the left eye had BRVO of 15 and 9 respectively. 
Table 3: Correlation Between Hypertension and Type of Occlusion

\begin{tabular}{|l|c|c|c|c|c|}
\hline Hypertension & ICRVO & NICRVO & HCRVO & BRVO & Total \\
\hline Yes & 5 & 3 & 2 & 12 & 22 \\
\hline No & 6 & 7 & 5 & 12 & 30 \\
\hline Total & 11 & 10 & 7 & 24 & 52 \\
\hline
\end{tabular}

It was observed that out of 52 patients, $22 \mathrm{had}$ hypertension and 30 were non hypertensive. Also noted that, out of 24 patients with BRVO, there were equal number of patients with and without hypertension and there was no statistically significant correlation ( $\mathrm{p}$ value of 0.618 ).

On analyzing the correlation between the types of occlusion with the presence of diabetes mellitus as a risk factor, it was observed that 17 patients had diabetes mellitus, while 35 patients had no history of diabetes. Among 17 Diabetics, 9 had BRVO, 4 had ICRVO, 2 each had NICRVO and HCRVO. Risk factor analysis showed statistically insignificant correlation with diabetes mellitus with a $\mathrm{p}$ value of 0.776 .

Fig 3.Correlation between Diabetes mellitus and Type of Venous Occlusion

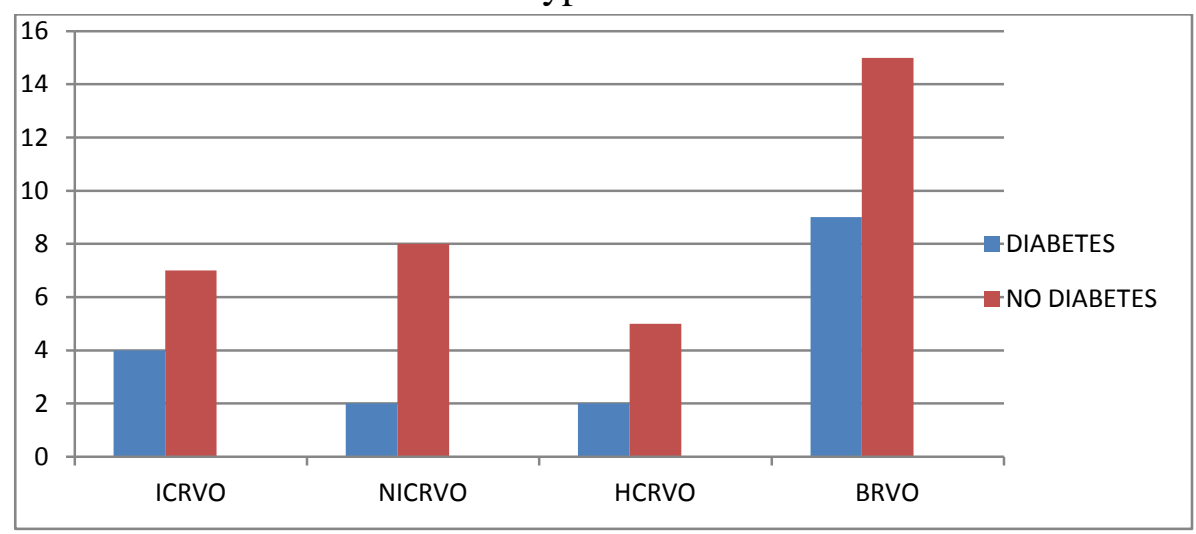

Table 4.Dyslipidemia and Type of Occlusions

\begin{tabular}{|l|c|c|c|c|c|}
\hline \multirow{2}{*}{ DYSLIPIDEMIA } & \multicolumn{4}{|c|}{ VENOUS OCCLUSION } & \multirow{2}{*}{ Total } \\
\cline { 2 - 5 } & ICRVO & NICRVO & HCRVO & BRVO & He \\
YES & 8 & 7 & 5 & 13 & 33 \\
NO & 3 & 3 & 2 & 11 & 19 \\
Total & 11 & 10 & 7 & 24 & 52 \\
\hline
\end{tabular}

High serum cholesterol value of more than $200 \mathrm{mg} / \mathrm{dl}$ was reported in 33 patients among which 13 were in the subclass of BRVO followed by 8 patients with ICRVO, 7 with NICRVO, 5 with HCRVO. Even though $63.46 \%$ patients had dyslipidemia, there was no statistically significant association with venous occlusion ( $\mathrm{p}$ value $=$ $0.642)$.
Fig 4 Hyperhomocysteinemia and type of venous occlusion

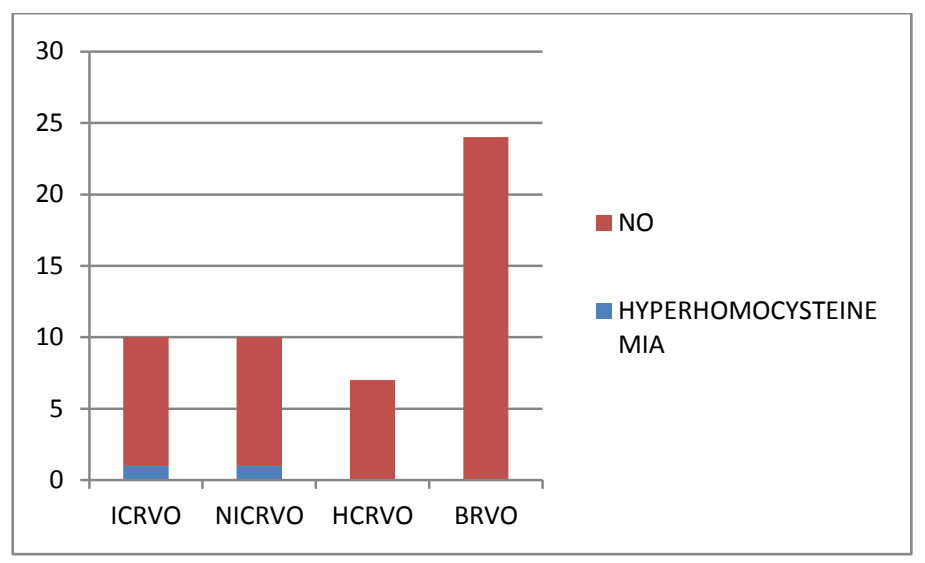


Table 5. Age group and Homocysteine

\begin{tabular}{|c|c|c|c|}
\hline \multirow{2}{*}{ Age Group in Years } & \multicolumn{2}{|c|}{ HOMOCYSTEINE } & \multirow{2}{*}{ Total } \\
\cline { 2 - 3 } & YES & NO & 1 \\
\hline $11-20$ & 1 & 0 & 4 \\
$21-30$ & 2 & 2 & 10 \\
$31-40$ & 0 & 10 & 37 \\
$41-50$ & 0 & 37 & 52 \\
Total & 3 & 49 & 5 \\
\hline
\end{tabular}

Presence of high levels of serum homocysteine was observed in 3 out of 52 patients, of which 2 belonged to age group of 21-30years and one in the age group of 11-20 years. This association showed a statistical significance with $\mathrm{p}$ value of $<0.001$.

Fig 5. Serum creatinine and type of venous occlusion

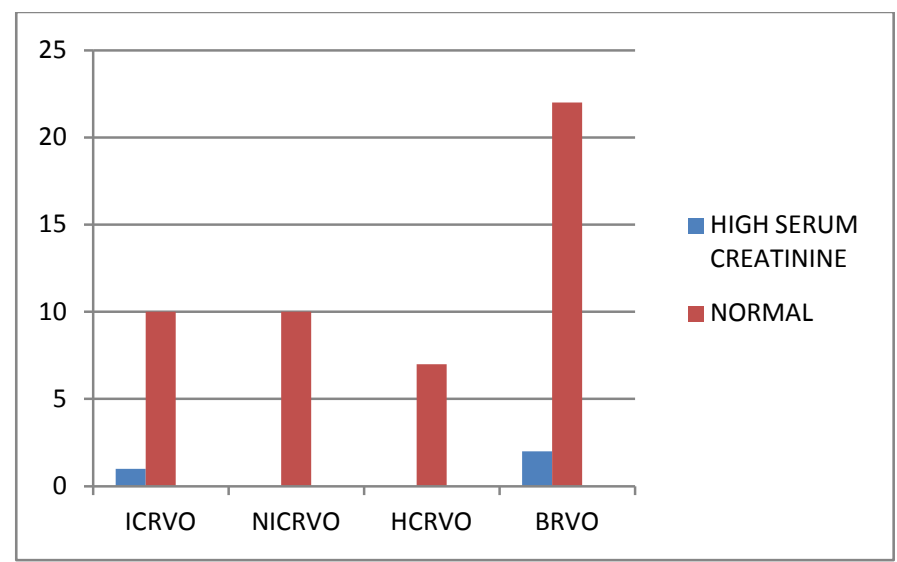

Three patients had abnormal serum creatinine value of which one of them had ischaemic CRVO, other two had BRVO.

Table 6 Correlation between smoking and venous occlusions

\begin{tabular}{|c|c|c|c|c|c|}
\hline \multirow{2}{*}{ Smoking } & \multicolumn{4}{|c|}{ TYPE OF OCCLUSION } & \multirow{2}{*}{} \\
\cline { 2 - 6 } & ICRVO & NICRVO & HCRVO & BRVO & Total \\
\hline YES & 1 & 1 & 1 & 5 & 8 \\
NO & 10 & 9 & 6 & 19 & 44 \\
Total & 11 & 10 & 7 & 24 & 52 \\
\hline
\end{tabular}

The majority of patients were nonsmokers (44) with presence of smoking as a risk factor only in 8 out of 52 patients and the association was not statistically significant.

Table 7. Correlation between vision and type of occlusions

\begin{tabular}{|c|c|c|c|c|c|c|}
\hline \multirow{2}{*}{ Type of RVO } & \multicolumn{5}{|c|}{ Vision } & \multirow[b]{2}{*}{ Total } \\
\hline & $6 / 9-6 / 12$ & $6 / 18-6 / 60$ & $5 / 60-2 / 60$ & 1/60-CFCF & HM-PL & \\
\hline ICRVO & 1 & 3 & 4 & 2 & 1 & 11 \\
\hline NICRV & 1 & 6 & 1 & 0 & 2 & 10 \\
\hline HCRVO & 2 & 3 & 1 & 1 & 0 & 7 \\
\hline BRVO & 2 & 16 & 3 & 3 & 0 & 24 \\
\hline Total & 6 & 28 & 9 & 6 & 3 & 52 \\
\hline
\end{tabular}

Visual acuity was stratified into 5 groups and 28 out of 52 were having a visual acuity of $6 / 18$ to 6/60, of which 16 patients had BRVO, 6 with NICRVO, 3 each in ICRVO and HCRVO. Severe visual loss of less than $1 / 60$ was observed in 9 patients, of whom 3 belonged to ischemic CRVO group with one patient having a vision of HM to PL category.

One patient had ocular risk factor of POAG, but no systemic risk factors, and one had ocular hypertension with additional systemic risk factors 
Table 8. Risk factors and number of patients

\begin{tabular}{|l|c|c|}
\hline Risk Factors & $\begin{array}{c}\text { Number of } \\
\text { patients }\end{array}$ & Percentage \\
\hline Age group 41-50 & 37 & 71.15 \\
\hline Dyslipidemia & 33 & 63.46 \\
\hline Hypertension & 22 & 42.30 \\
\hline Diabetes Mellitus & 17 & 32.69 \\
\hline Smoking & 8 & 15.38 \\
\hline Hyper homocysteinemia & 3 & 5.76 \\
\hline High Serum creatinine & 3 & 5.76 \\
\hline POAG,OHT & 2 & 3.85 \\
\hline Multiple risk factors & 21 & 40.38 \\
\hline No risk factors & 11 & 21.15 \\
\hline
\end{tabular}

\section{Discussion}

The various metabolic risk factors and its association with RVO were analyzed. The patients studied in the sample were from 16 to 50 years of age with mean age of 38.45. Out of 52 patients, 29 $(56 \%)$ were males and $23(42 \%)$ females. In a study conducted in India by Prajapathi et al in 2014 showed that the prevalence of retinal vein occlusion was more among males ${ }^{(5)}$. As most of the current studies were based on older age groups, we highlighted on the incidence of venous occlusions in younger age group up to 50 years with majority $(71 \%)$ of cases in the 41 to 50 years. In a Similar study among younger patients by Rechhia Franco M et al published in 2004 ,the mean group was 45 years and $55 \%$ were males, similar to our study ${ }^{(6)}$.

On assessing the laterality of occurrence of venous occlusions, it was observed that 30 were in the right eye and 22 in the left eye. Similar result was obtained by Prajapathi et al. Of the 52 patients with retinal venous occlusion, they were divided into 4 groups with 11 patients in ischemic CRVO, 10 nonischmeic,7 hemi CRVO and 24 BRVO. Among patients with BRVO, majority (58\%) had supero temporal branch vein occlusion. As far as overall profile of venous occlusion was concerned, in this study 33 of them had hypercholesterolemia, 22 out of the total 52 patients were hypertensive and 17 were having diabetes mellitus. It was observed that metabolic risk factors like dyslipidemia, hypertension and diabetes mellitus were in the forefront. But in contrary to the above observation, analysis on the risk factors for younger patients with venous occlusions showed that correlation between these factors were statistically not significant. Further we did not find hypertension as a confounding factor for the development of RVO. Hypertension was known to cause atherosclerosis and linked to their age; hence this may explain the lower incidence of hypertension in young venous occlusions as the incidence of atherosclerosis is less in this group. Similar study in young Chinese population by Jane et al also showed that, at presentation majority of the patients had no identifiable systemic risk factors. It was also observed in the study that $91 \%$ of these patients on follow up developed risk factors like hypertension and diabetes mellitus ${ }^{(7)}$.

Hyperlipidemia was observed in 33 subjects and this finding strongly implicates the need for evaluation for dyslipidemia in all cases and need for control of blood lipid levels to prevent the occurrence of RVO. Hence it is necessary that thorough systemic evaluation has to be carried out in patients presenting with venous occlusions and prompt follow up be ensured since their likelihood of developing metabolic risk factors is significantly high. So early identification can control and prevent life threatening systemic complications to which they are prone to.

Homocysteine is a sulfur amino acid whose metabolism is affected by the deficiency of vitamins like folic $\operatorname{acid}^{(8)}$. Serum levels of homocysteine has shown to have significant influence on the development of venous occlusions especially in the younger age groups. High homocysteine plasma levels have been associated with cardiovascular disease, deep vein thrombosis and pulmonary embolism, stroke, and abdominal aortic aneurysm ${ }^{(9,10,11)}$. In our sample, 3 patients were having high serum levels and they belonged to age group of less than 30 years. In an Iranian study by Moghimi et al in 2008, it was concluded that elevated total homocysteine level was an independent risk factor for $\mathrm{CRVO}^{(13)}$ and measurement of serum levels should be made a part of initial investigation especially in younger 
patients with no identifiable systemic risk factors. Correction of homocysteine levels with vitamin supplementation in order to prevent recurrence of the disease in either eye has also been studied ${ }^{(12)}$. Cigarette smoking as a risk factor for retinal venous occlusions has been proven by Hayreh ${ }^{(14)}$, but in our group, no statistical significance was obtained with smoking, only 8 out of 52 were smokers. This could be attributable to the lower age group we have chosen as the harmful vascular damage by smoking becomes evident as the age advances and the toxin accumulates in the due course of continued and prolonged smoking.

Of the ocular risk factor analysed, one patient had primary open angle glaucoma (POAG), but no systemic risk factors, and one had ocular hypertension with additional systemic risk factors of diabetes, hypertension and dyslipidemia, both of them had BRVO. There was no systemic or ocular risk factor to account for venous occlusion in 11 patients, of which 8 of them had BRVO, 2 had HCRVO and 1 had NICRVO. None of them had Ischemic type of CRVO.

\section{Conclusion}

Retinal vein occlusion in the young may precede various life threatening systemic diseases and it can be considered as a precursor to unveil their occurrence. Association of retinal venous occlusion with hyper homocysteinemia was found to be significant in this age group and can be treated with vitamin supplementation to prevent any future recurrence or systemic threats. Dyslipidemia was attributed to be a major modifiable risk for development of venous occlusion even in the young. As multiple risk factors were associated in many cases, a detailed examination and thorough search for the underlying risk is warranted in cases of venous occlusion especially in the young population and all of them should be followed up for the development of both ocular and systemic complications in future.

\section{Limitations}

This was a cross sectional study where the data regarding the systemic risk factors and investigations were collected at the initial period of diagnosis. Hence follow up of the patient for the occurrence of any new risk factor could not be included in this sample. Though patients were identified with established metabolic diseases like diabetes and hypertension, we could not quantify the level of blood sugar values or exact blood pressure range at which RVO occurred as the patient included were within one month of diagnosis and their status of metabolic control could not be judged from this study.

\section{Sources of Support: Nil}

\section{References}

1. Mitchell P, Smith W, Chang A. Prevalence and associations of retinal vein occlusion in Australia: the Blue Mountains Eye Study. Arch Ophthalmol.1996;114:1243-7

2. Hayreh SS, Zimmerman B, McCarthy MJ, Podhajsky P. Systemic diseases associated with various types of retinal vein occlusion. Am J Ophthalmol. 2001; $131(1: 61-77$

3. Martin SC, Butcher A, Martin N, Farmer J, Dobson PM, Bartlett WA, et al. Cardiovascular risk assessment in patients with retinal vein occlusion. $\mathrm{Br} \mathrm{J}$ Ophthalmol. 2002;86 (7:774-776.

4. Sharma R, Bhat MA. Risk Factors in Retinal Vein Occlusion.

5. Prajapati VA, Vasavada D, Patel SM, Chauhan W, Prajapati V. A study of evaluation of various risk factors of retinal vein occlusion.International Journal of Research in Medical Sciences. 2017 Jan 24;2(3):1054-7.

6. Recchia FM, Brown GC. Systemic disorders associated with retinal vascular occlusion. Current opinion in ophthalmology. 2000 Dec 1;11(6):462-7. 
7. Kuo JZ, Lai CC, Ong FS, Shih CP, Yeung L, Chen TL, Chen KJ, Wu WC. Central retinal vein occlusion in a young Chinese population: risk factors and associated morbidity and mortality. Retina (Philadelphia, Pa.). 2010 Mar;30(3):479.

8. Homocysteine Studies Collaboration. Homocysteine and risk of ischemic heart disease and stroke: A metaanalysis. JAMA. 2002;288:2015-22.

9. Casas JP, Bautista LE, Smeeth L, Sharma P, Hingorani AD. Homocysteine and stroke: Evidence on a causal link from mendelianrandomisation. Lancet. 2005;36 5:224-32.

10. Takagi H, Umemoto T. Homocysteinemia is a risk factor for aortic dissection. Med Hypotheses. 2005;64:1007-10.

11. Giusti B, Marcucci R, Lapini I, Sestini I, Lenti M, Yacoub $M$, et al. Role of hyperhomocysteinemia in aortic disease. Cell MolBiol (Noisy-legrand) 2004;50:945-52.

12. Moghimi S, Najmi Z, Faghihi H, Karkhaneh R, Farahvash MS, Maghsoudipour M. Hyperhomocysteinemia and central retinal vein occlusion in Iranian population. International ophthalmology. 2008 Feb 1;28(1):23-8.

13. Eye Disease Case-Control Study Group. Risk factors for branch retinal vein occlusion. American journal of ophthalmology. 1993 Sep 1;116(3):286-96

14. Hayreh SS. Ocular vascular occlusive disorders: natural history of visual outcome. Progress in retinal and eye research. $2014 \mathrm{Jul}$ 31;41:1-25. 